\title{
Incidence and predictors of left ventricular thrombus by cardiovascular magnetic resonance in acute ST-segment elevation myocardial infarction treated by primary percutaneous coronary intervention: a meta-analysis
}

Heerajnarain Bulluck ${ }^{1,2^{*}}$ D, Mervyn H. H. Chan ${ }^{3,6}$, Valeria Paradies ${ }^{3}$, Robert L. Yellon ${ }^{2}, \mathrm{He}$ H. Ho ${ }^{4}$, Mark Y. Chan ${ }^{5}$, Calvin W. L. Chin ${ }^{6}$, Jack W. Tan ${ }^{6}$ and Derek J. Hausenloy ${ }^{2,3,7,8,9,10}$

\begin{abstract}
Introduction: The incidence of left ventricular (LV) thrombus formation in ST-segment elevation myocardial infarction (STEMI) patients in the current era of primary percutaneous coronary intervention (PCI) is not well established. We performed a meta-analysis to assess the actual incidence and predictors of LV thrombus by cardiovascular magnetic resonance (CMR) in STEMI treated by primary $\mathrm{PCl}$.

Methods: We searched MEDLINE and EMBASE databases up to February 2018. We included all studies published as a full-text article, reporting the incidence of LV thrombus by CMR within 1 month following acute STEMI in patients treated by primary $\mathrm{PCl}$. A binary random-effects model was used to estimate the pooled incidence of LV thrombus. The diagnostic performance of transthoracic echocardiography (TTE) as compared with CMR was pooled to obtain the sensitivity and specificity of TTE with CMR as the gold standard. Embolic and bleeding complications of LV thrombus were also evaluated.
\end{abstract}

Results: Ten studies were included in the meta-analysis. The incidence of LV thrombus by CMR in all-comer STEMI patients ( $n=2072$ ) was $6.3 \%$ with $96 \%$ of LV thrombus occurring in those with anterior STEMI (12.2\% incidence). When only anterior STEMI with LVEF $<50 \%$ were considered $(n=447)$, the incidence of $L V$ thrombus was $19.2 \%$. Compared with CMR, the sensitivity of TTE to detect LV thrombus was $29 \%$ with a specificity of $98 \%$. The sensitivity of TTE increased to $70 \%$ in those with anterior STEMI and reduced LVEF. LV thrombus resolved in $88 \%$ of cases by 3 to 6 months. After $1-2$ years follow-up, the embolic complication rate was similar at $1.5 \%(P=0.25)$ but the bleeding complication rate was significantly higher $(8.8 \%$ versus $0.5 \%, P<0.001)$ in the LV thrombus group on triple therapy when compared to the no LV thrombus group on dual antiplatelet therapy.

(Continued on next page)

\footnotetext{
* Correspondence: h.bulluck@gmail.com

${ }^{1}$ Norfolk and Norwich University Hospital, Colney Lane, Norwich NR4 7UY, UK

${ }^{2}$ The Hatter Cardiovascular Institute, Institute of Cardiovascular Science,

University College London, London, UK

Full list of author information is available at the end of the article
}

(c) The Author(s). 2018 Open Access This article is distributed under the terms of the Creative Commons Attribution 4.0 International License (http://creativecommons.org/licenses/by/4.0/), which permits unrestricted use, distribution, and reproduction in any medium, provided you give appropriate credit to the original author(s) and the source, provide a link to the Creative Commons license, and indicate if changes were made. The Creative Commons Public Domain Dedication waiver (http://creativecommons.org/publicdomain/zero/1.0/) applies to the data made available in this article, unless otherwise stated. 


\begin{abstract}
(Continued from previous page)
Conclusion: In the primary PCl era, CMR detection of an LV thrombus post-STEMI remains high with incidence of nearly

20\% in anterior STEMI with depressed LVEF. Patients with LV thrombus treated by triple therapy had similar embolic

complications but higher bleeding complications than those with no LV thrombus treated with dual antiplatelet therapy.

A 3 month follow-up CMR scan to guide anticoagulation duration might help mitigate bleeding risk.
\end{abstract}

Keywords: Left ventricular thrombus, ST-segment elevation myocardial infarction, Cardiovascular magnetic resonance, Primary percutaneous coronary intervention

\section{Background}

Primary percutaneous coronary intervention (PCI) is the reperfusion strategy of choice for the treatment of ST-segment elevation myocardial infarction (STEMI). Although left ventricular (LV) thrombus formation is a recognized complication in STEMI patients, its incidence in the era of primary PCI is not well established. LV thrombus may lead to embolic complications such as stroke, with devastating consequences. Both the American College of Cardiology/American Heart Association/and European Society of Cardiology guidelines $[1,2]$ recommend a minimum of 3 to 6 months of anticoagulation (Class IIb and IIa respectively, level of evidence $C$ ) with subsequent repeated imaging to guide ongoing anticoagulation.

A meta-analysis recently reported that the ovrall rate of LV thrombus by transthoracic echocardiography (TTE) in the primary PCI for STEMI era was $2.7 \%$ with 9.1\% for anterior STEMI [3]. However, TTE has a lower sensitivity (35\%) for detecting LV thrombus when compared to cardiovascular magnetic resonance (CMR) [4]. However, TTE relies on the morphological identification of LV thrombus, whereas CMR with gadolinium contrast agent can identify LV thrombus based on both its morphology and tissue characteristics [4].

We therefore performed a meta-analysis to assess the incidence and predictors of LV thrombus by CMR in STEMI patients treated by primary PCI in the current era and to evaluate how it may be used in the clinical setting, in conjunction with TTE, to improve LV thrombus detection.

\section{Methods}

This meta-analysis adhered to the Preferred Reporting Items for Systematic Reviews and Meta-Analyses (PRISMA) statement [5] and was performed according to the recommendations specified in the Cochrane Handbook for Systematic Reviews of Interventions [6].

\section{Eligibility criteria}

All studies reporting on the incidence of LV thrombus by CMR in STEMI patients treated by primary PCI were eligible for inclusion. The inclusion criteria were all studies published as a full-text article reporting the CMR incidence of LV thrombus within 1 month following acute STEMI in patients treated by primary PCI. Conference abstracts were excluded.

\section{Search strategy}

We searched Ovid MEDLINE and Ovid EMBASE databases up to February 2018. Furthermore, we screened editorials and references of eligible studies. The following search terms were used: "ventricular", "apical", "thrombus", "magnetic resonance imaging", "acute myocardial infarction", "primary percutaneous intervention".

\section{Study selection}

Two authors (HB, MHHC) identified suitable articles independently. Disagreement was resolved through consensus from a third investigator (DJH). Figure 1 shows the process of study selection as per PRISMA [5].

\section{Data extraction and quality assessment}

Baseline clinical characteristics of the study population were extracted. Trial quality was determined as per the Cochrane Handbook [6] but without constructing a composite quality score given the limitations inherent to such an approach [7]. We aimed to produce a funnel plot if there were $>10$ included studies in the forest plots.

\section{Endpoints}

The main endpoints analyzed were incidence of LV thrombus identified on the acute CMR and follow-up CMR when available. Furthermore, the incidences of embolic and bleeding complications as defined by the individual studies were also collected.

\section{Statistical analysis}

The studies were divided into those including all-comer STEMI, anterior STEMI patients and those with anterior STEMI and with reduced LV ejection fraction (LVEF). A binary random-effects model was used to estimate the pooled incidence of LV thrombus with 95\% confidence interval (CI) using OpenMeta[Analyst] [8] software. Where available, studies 


\section{PRISMA 2009 Flow Diagram}

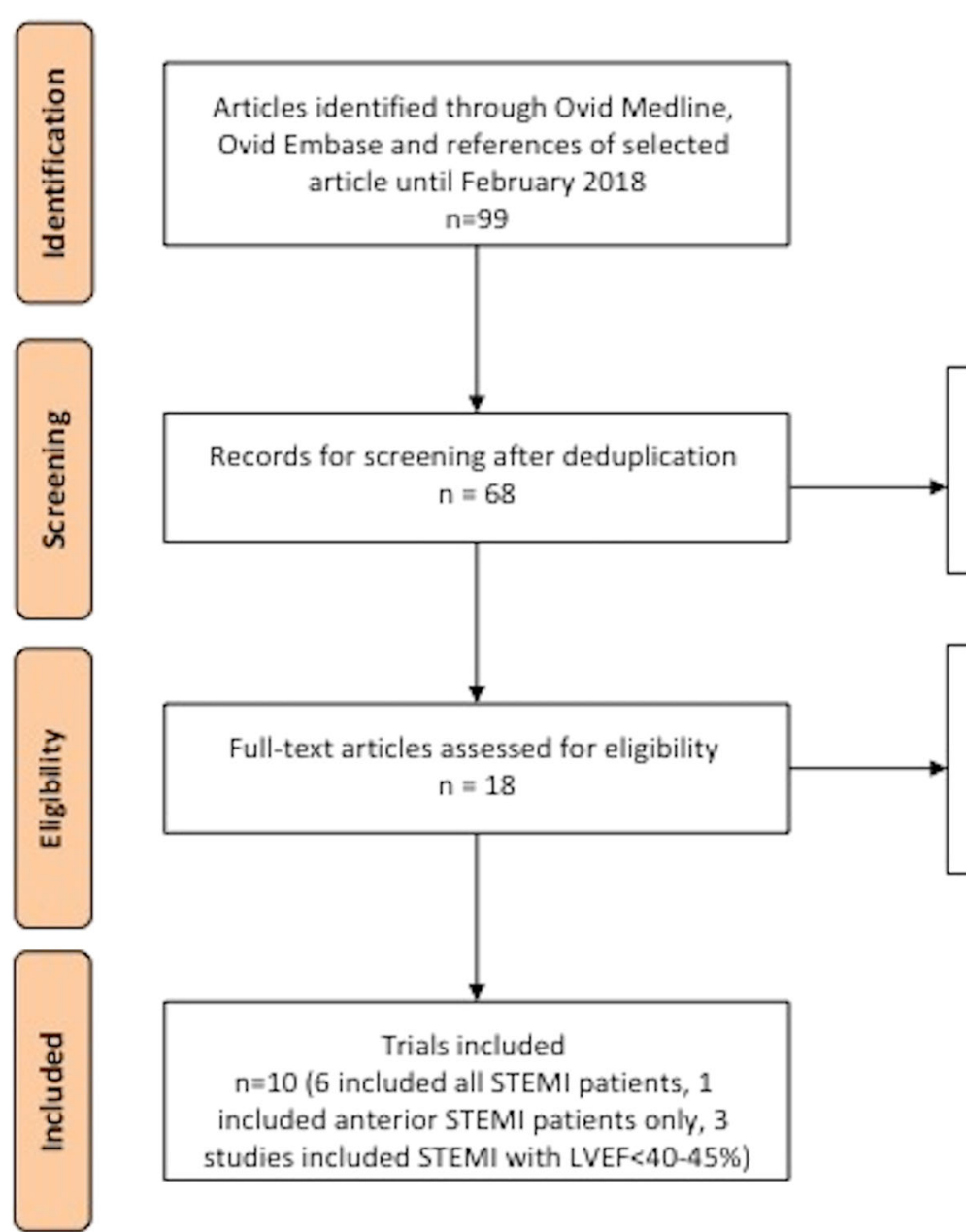

$n=42$, records excluded as clearly not relevant on checking titles and abstracts

8 - conference abstracts only

Reasons for excluding full-text articles

3 - included chronic MI

1 - LVT by either CMR or echo

4 - review articles

Fig. 1 PRISMA flow diagram of the study selection process. This is the PRISMA flow diagram showing how studies were identified, screening and included in this meta-analysis

comparing the diagnostic performance of TTE versus CMR were pooled to obtain the sensitivity and specificity of TTE against CMR. Lastly, embolic complications (ischemic stroke and clinically significant peripheral embolisation) and bleeding complications between those with LV thrombus against those without LV thrombus were also compared. Heterogeneity among the studies included in each forest plot was quantified using $\mathrm{I}^{2}$ statistics with $\mathrm{I}^{2}$ of $0-25 \%, 25-50 \%$ and $50-75 \%$ considered as low, moderate and high heterogeneity, respectively.

\section{Results}

Ten studies [4, 9-17] were included in the meta-analysis. The characteristics of these studies are summarized in Table 1. The CMR scan was performed within the first week post-primary PCI in 7 studies [10-16], and between 7 and 30 days in 3 studies $[4,9,17]$.

\section{Incidence of LV thrombus}

Six studies [4, 10-14] included data on LV thrombus in anterior versus non-anterior STEMI. The overall incidence of LV thrombus in STEMI patients $(n=2072)$ was $6.3 \%$ (95\%CI $4.2-8.5), \mathrm{I}^{2}$ of $72 \%$ (Fig. 2) with $96 \%$ (111/ 116) of thrombi occurring with anterior STEMI. Among those with anterior STEMI only (7 studies, $n=1244)$ [4, 9-14], the incidence of LV thrombus was $12.2 \%$ (95\%CI 9.0-15.4\%), $\mathrm{I}^{2}=64 \%$, (Fig. 2).

Sensitivity analysis was performed by excluding the studies by Poss 2015 [10] and Lanzillo 2013 [11] (outliers on the Forest plot in Fig. 1). The overall incidence of LV thrombus was similar at $6.5 \%(95 \% \mathrm{CI} 5.0-7.9 \%)$ STEMI 


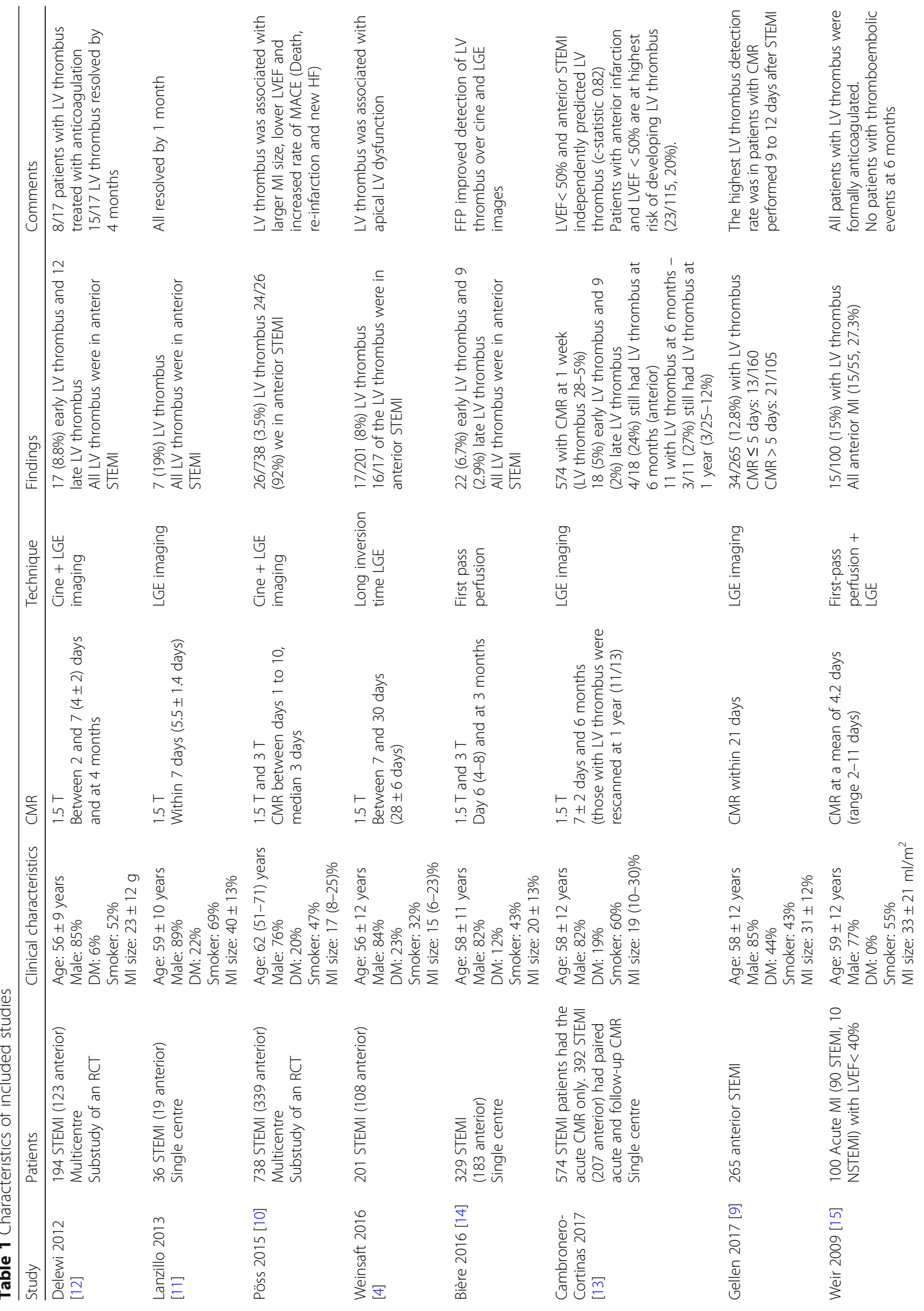




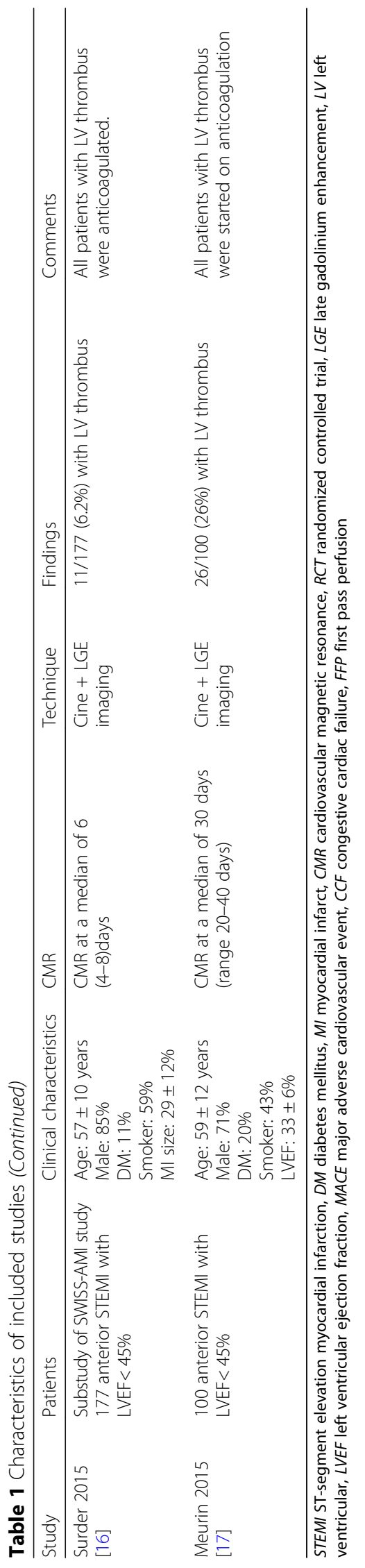




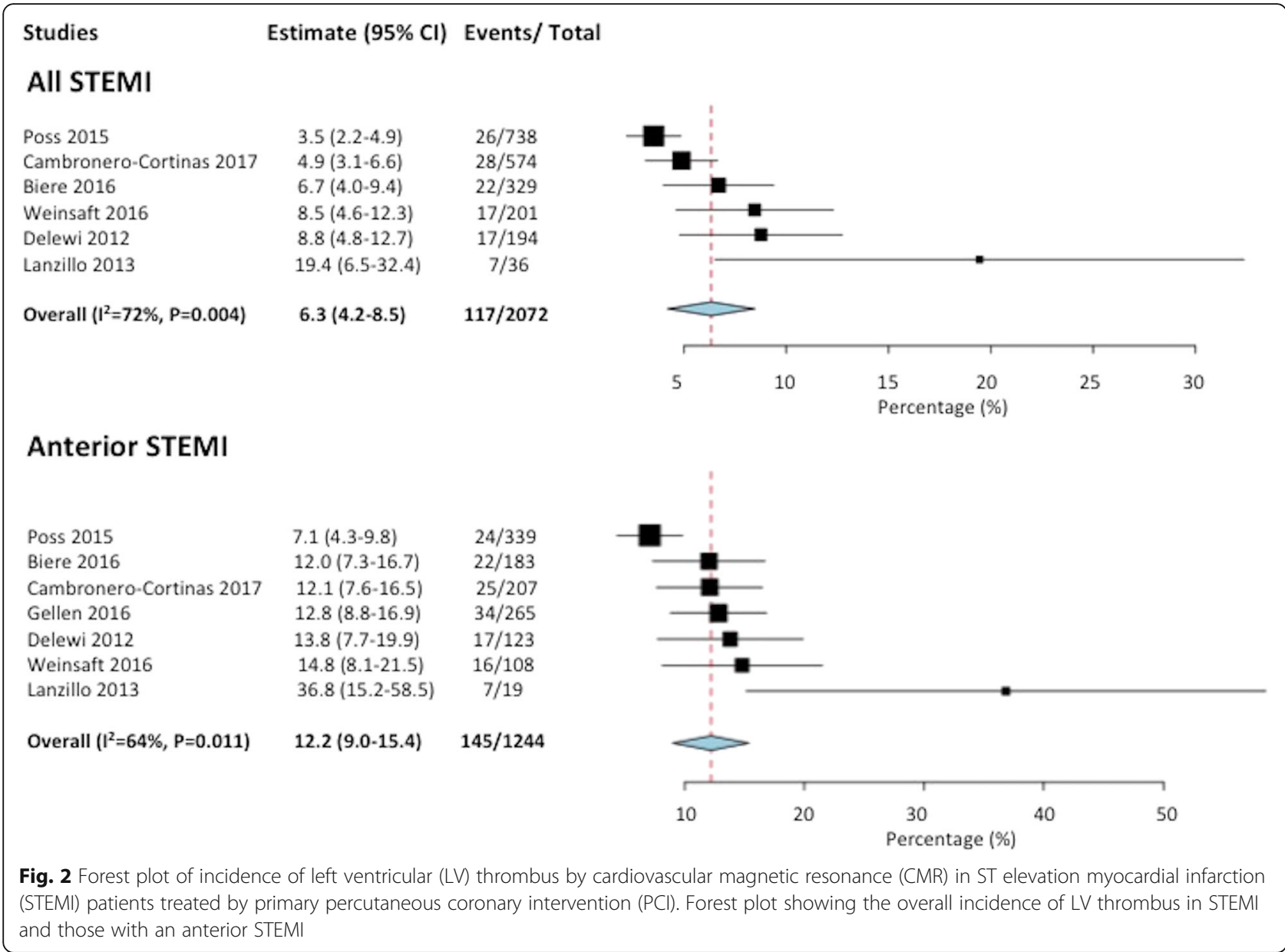

$(n=1298)$ and was $12.8 \%$ (95\%CI $10.6-15.0 \%)$ for anterior STEMI $(n=886)$, with $\mathrm{I}^{2}$ of $9 \%$ and $0 \%$, respectively.

Three studies [15-17] included only patients with LVEF $<40-45 \%$. One study [13] also reported the incidence of LV thrombus in those with anterior STEMI and $\mathrm{LVEF}<50 \%$. When only anterior STEMI with reduced LVEF were considered, the pooled incidence of LV thrombus in the 447 patients was $19.2 \%$ (95\%CI 7.7$30.8 \%), \mathrm{I}^{2}=90 \%$.

Three studies (acute CMR performed within the first week) also had a repeat CMR between 3 to 6 months [1214]. LV thrombus resolved in $88 \%$ (50/57) of cases. However, late LV thrombus was detected in an additional 3.2\% (95\%CI $1.4-5.1 \%)$ of cases $(29 / 868)$ on the 3 to 6 months CMR.

\section{Sensitivity and specificity of TTE against CMR}

Three studies $[4,11,12]$ presented data on LV thrombus detection by both TTE and CMR. Among the 431 patients with both CMR and TTE data, using CMR as the reference standard, the sensitivity of TTE was only $29 \%$ (95\%CI $17-$ $45 \%)$ with a specificity of $98 \%$ (95\%CI $96-99 \%)$.
For anterior STEMI with reduced LVEF, 3 studies also presented data on the detection of LV thrombus by both TTE and CMR. For the 246 patients, the sensitivity of TTE for LV thrombus was 70\% (95\%CI 56-82\%) with a specificity of 98\% (95\%CI 94-99\%).

\section{Embolic and bleeding complications}

Three studies $[12,13,17]$ reported embolic and bleeding complications separately. All patients with LV thrombus were started on triple therapy (anticoagulation together with dual antiplatelet therapy).

After a follow-up period of between 1 and 2 years, the incidence of embolic complications (ischemic stroke and distal embolisation) was similar at $1.5 \%$ in both the LV thrombus group (1/68) on triple therapy (anticoagulation plus dual antiplatelet therapy) and no LV thrombus group (9/616) on dual antiplatelet therapy only, $P=0.25$, $\mathrm{I}^{2}=0 \%$. However, bleeding complications were significantly higher in the LV thrombus group $(6 / 68,8.8 \%)$ than in the no LV thrombus group (3/616, 0.5\%), $P<$ $0.001, \mathrm{I}^{2}=65 \%$. 


\section{Discussion}

In this meta-analysis of more than 2000 patients, overall CMR evidence of LV thrombus was $>6 \%$ of primary PCI STEMI patients. This is more than twice that reported by TTE [3]. We also confirmed that TTE had a poor sensitivity of only $29 \%$ when compared to CMR. The strength of CMR lies in its spatial resolution for morphological definition of the LV thrombus. Avascular thrombus can also be characterized and differentiated from neighbouring structures using gadolinium chelate contrast as illustrated in Fig. 3.

Expectedly, the large majority (96\%) of LV thrombus by CMR occurred in those with anterior STEMI. The incidence of LV thrombus in anterior STEMI was more than $12 \%$. In the subgroup of patients with anterior STEMI and LVEF $<50 \%$, LV thrombus by CMR was detected in a remarkably high proportion of patients: one in five patients. Of note, the sensitivity of TTE was higher at $70 \%$ in this subgroup of patients.

The incidence of LV thrombus in the study Poss 2015 [10] was lowest (3.5\%), despite including the largest number of patients $(n=738)$. This may be related to the early use of the potent glycoprotein IIb/IIIa inhibitor abciximab (intracoronary or intravenous) and warrants further investigation.

The incidence of LV thrombus by CMR is dependent on the timing of the CMR scan. An additional 3\% of patients were identified to have late LV thrombus of the CMR performed between 3 and 6 months. Gellen et al. [9] recently showed that the highest LV thrombus detection rate (25\%) was in those with CMR performed 9-12 days after anterior STEMI and Meurin et al. [17] showed a similar finding with those having the CMR scan between 8 and 15 days having the higher incidence of LV thrombus. These data suggest that the optimal timing of imaging for LV thrombus following an acute STEMI may be at 2 weeks.

Consistent with prior studies, risk factors for LV thrombus formation were anterior STEMI and LVEF<
$50 \%[10,13]$. The presence of microvascular obstruction (MVO) [10] and apical wall motion abnormality [4] has also been associated with the development of $\mathrm{LV}$ thrombus. It has also recently been shown that LV thrombus was associated with a composite of heart failure, re-infarction and mortality [10]. This is likely a reflection of those with LV thrombus having larger infarct size, higher burden of MVO and lower LVEF.

Although the number of studies was limited, accounting for 684 patients, the incidence of embolic complications in those with LV thrombus and treated by anticoagulation was similar to those without LV thrombus. However, as expected, those with LV thrombus and triple therapy had higher incidence of bleeding complications. The large proportion of LV thrombus (88\%) resolved on the repeat CMR performed between 3 to 6 months. A follow-up CMR scan as early as 3 months after STEMI may reduce the need for prolonged anticoagulation in a proportion of patients.

LV thrombus post acute STEMI is well recognised to occur predominantly at the apex [4] and in those with reduced LVEF [13]. The likely explanation why the incidence of LV thrombus is higher in anterior STEMI is likely because the left anterior descending artery subtends the largest amount of myocardium compared with the left circumflex coronary artery and right coronary artery and the left anterior descending artery wraps around the apex in three quarter of cases [18]. Moreover, a large anterior STEMI involving the apex would lead to blood stasis and endothelial injury, pre-requisites for the development of LV thrombus [19]. Despite the low sensitivity of TTE, it is more widely available and more affordable than CMR. Apical wall motion score has been shown to be useful to identify those would benefit from CMR imaging for LV thrombus detection. Therefore, we propose an algorithm, starting from the time of primary PCI that may be used to identify those most likely to develop LV thrombus and who may benefit from CMR if TTE is inconclusive (Fig. 4).

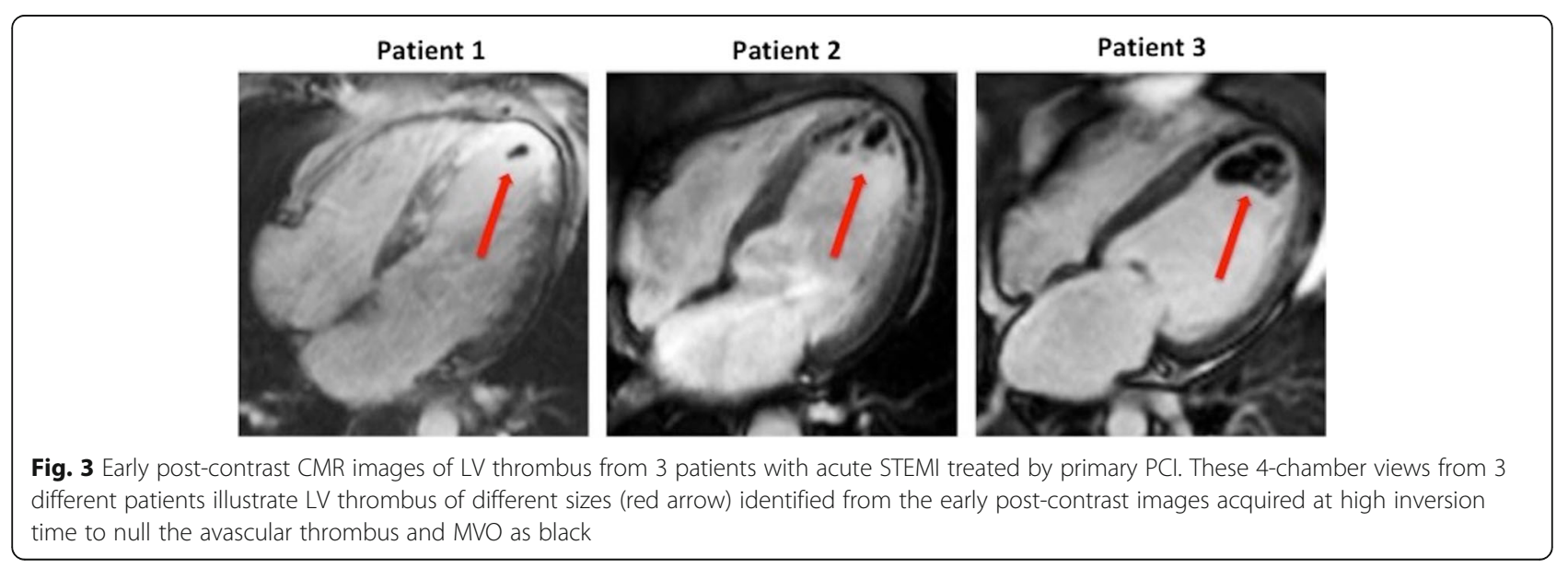




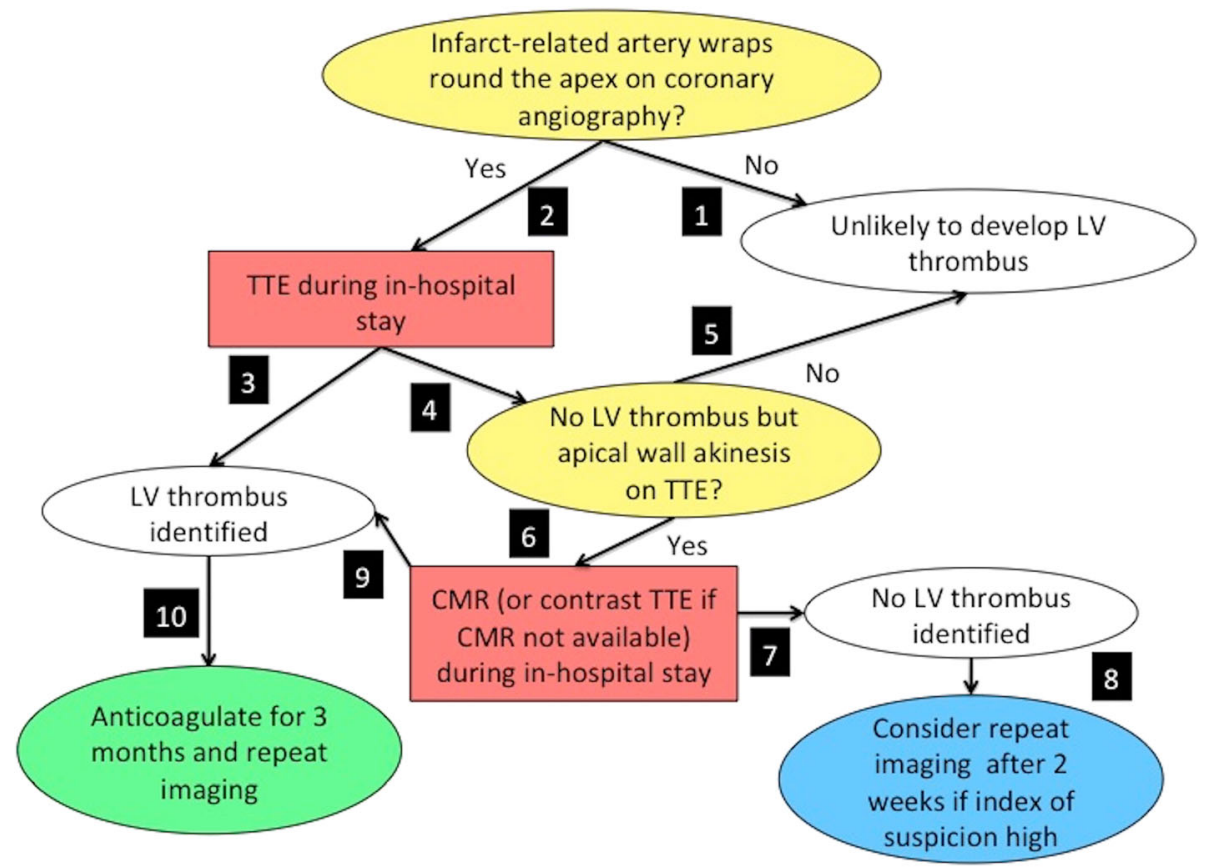

Fig. 4 Algorithm for the detection of LV thrombus in reperfused STEMI patients in the clinical setting. This algorithm provides guidance on how STEMI patients at risk could be identified and how TTE and CMR could be integrated in the clinical setting to optimize the detection of LV thrombus

LV thrombus can sometimes be difficult to distinguish from MVO. The following previously described criteria [20] can be applied to differentiate between the two on late gadolinium enhanced images: location (LV thrombus tends to be intra-cavity whereas $\mathrm{MVO}$ is intra-myocardial); contrast fill-in on subsequent late gadolinium enhanced images would occur in the context of MVO but not with LV thrombus; differences in appearance (LV thrombus is usually well-defined with sharp borders whereas MVO tends to be patchy and inhomogeneous) as shown in Fig. 5.

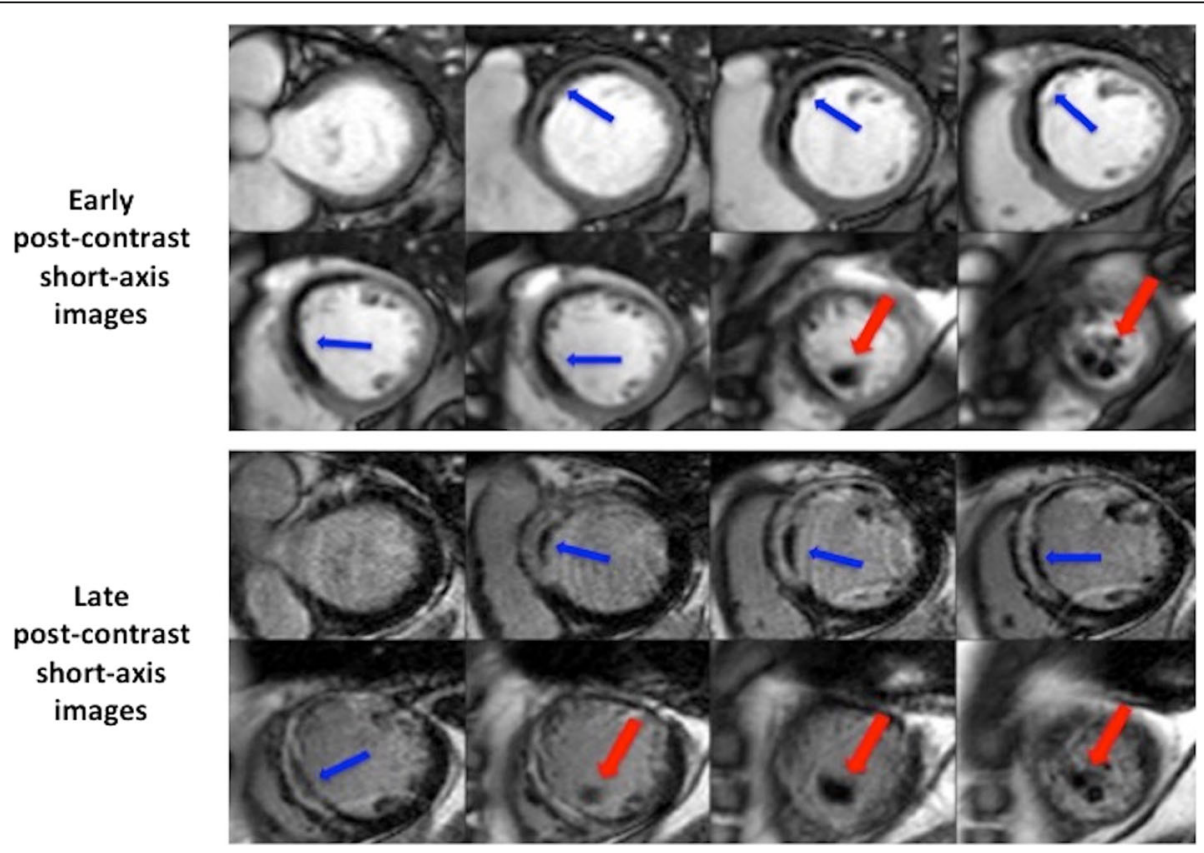

Fig. 5 Differences in appearance between LV thrombus and microvascular obstruction (MVO). These are the early and late post-contrast short-axis images from a patient with LV thrombus and MVO. The red arrows show the LV thrombus and the blue arrows delineate the MVO 
Contrast TTE has also been used for the detection of LV thrombus with an improved sensitivity of $64 \%$ when compared to CMR [4]. This suggests that CMR may still be useful in high risk patients despite a negative contrast TTE.

In the chronic MI setting, in a cohort of patients with ischemic heart disease undergoing assessment for LV reconstruction surgery, the sensitivity of CMR against the pathological confirmation of LV thrombus was high at 88\% [21]. The sensitivity of non-simultaneous TTE in that study was as low as $23 \%$ and that of intraoperative transesophageal echocardiography was only $40 \%$ [21]. The finding from our study is in line with this previous study showing a poor sensitivity of TTE to detect LV thrombus.

\section{Limitations}

The techniques used in this meta-analysis to detect LV thrombus by TTE did not include echo contrast or newer 3D volumetric methods. The techniques used to detect LV thrombus by CMR (first-pass perfusion [14], conventional late gadolinium enhancement [9-13] or long inversion time gadolinium enhancement imaging [4]) and the timing of CMR in relation to the primary PCI were not standardized in the included studies. The studies were included in the forest plots showed high heterogeneity but we performed sensitivity analysis and this showed consistent results while improving the $\mathrm{I}^{2}$ significantly. Several of the secondary analyses included data from only 3 studies, but these constituted the largest pooled analyses and need to be confirmed in future larger prospective studies. Patient-level data were not available. Despite these limitations, our study provides a more accurate estimate of the true incidence of $\mathrm{LV}$ thrombus from a pooled analysis of $>2000$ STEMI patients in the current primary PCI era. However, a proportion of STEMI patients with LV thrombus currently go undetected, as CMR is not part of routine practice. The natural history of these patients, especially in the current era of potent antiplatelet therapy is not known and could not be answered by this study. Lastly, CMR is currently not widely available, is relatively expensive and there may be an element of selection bias (only patients fit enough to lie flat, with no contraindications would tolerate the scan).

\section{Conclusion}

CMR is a valuable tool for the detection of LV thrombus post-primary PCI for acute STEMI and the incidence remains clinically substantial at $6 \%$ of all-comers STEMI, $12 \%$ of anterior STEMI and 19\% of anterior STEMI patients with reduced LVEF. We propose that CMR may have a role in high-risk STEMI patients (akinetic apex) and in patients with inconclusive TTE. Patients with LV thrombus treated with triple therapy (anticoagulation and dual antiplatelet therapy) have similar embolic complication rates but higher bleeding complication rates as compared with those with no LV thrombus treated with dual antiplatelet therapy only. We suggest a repeat CMR scan at 3 months post-STEMI to guide anticoagulation duration and to potentially reduce bleeding risk.

\section{Abbreviations \\ Cl: Confidence interval; CMR: Cardiovascular magnetic resonance; EF: Ejection fraction; LV: Left ventricle/left ventricular; MVO: Microvascular obstruction; PCI: Percutaneous coronary intervention; PRISMA: Preferred Reporting Items for Systematic Reviews and Meta-Analyses; STEMI: ST-segment elevation myocardial infarction; TTE: Transthoracic echocardiography}

\section{Funding}

This work was supported in part by grants from the British Heart Foundation (FS/10/039/28270), the National Institute for Health Research University College London Hospitals Biomedical Research Centre, Duke-National University Singapore Medical School; the Singapore Ministry of Health's National Medical Research Council under its Clinician Scientist-Senior Investigator scheme (NMRC/CSA-SI/0011/2017) and Collaborative Centre Grant scheme (NMRC/CGAug16C006), the Singapore Ministry of Education Academic Research Fund Tier 2 (MOE2016-T2-2-021), and the EU-CARDIOPROTECTION CA16225 Cooperation in Science and Technology Action to Prof Hausenloy.

Availability of data and materials

Further details on the data used to reach the conclusions in this manuscript are available upon request.

\section{Authors' contributions}

Made substantial contributions to conception and design, or acquisition of data, or analysis and interpretation of data: $\mathrm{HB}, \mathrm{MCHH}, \mathrm{VP}, \mathrm{RLY}, \mathrm{DJH}$. Been involved in drafting the manuscript or revising it critically for important intellectual content: HB, HHH, MYC, CWLC, JWT, DJH. All authors have given final approval of the version to be published and agree to be accountable for all aspects of the work in ensuring that questions related to the accuracy or integrity of any part of the work are appropriately investigated and resolved.

Ethics approval and consent to participate

Not applicable.

Consent for publication

Not applicable.

\section{Competing interests}

The authors declare that they have no competing interests.

\section{Publisher's Note}

Springer Nature remains neutral with regard to jurisdictional claims in published maps and institutional affiliations.

\footnotetext{
Author details

'Norfolk and Norwich University Hospital, Colney Lane, Norwich NR4 7UY, UK. ${ }^{2}$ The Hatter Cardiovascular Institute, Institute of Cardiovascular Science, University College London, London, UK. ${ }^{3}$ National Heart Research Institute Singapore, National Heart Centre Singapore, Singapore, Singapore.

${ }^{4}$ Department of Cardiology, Tan Tock Seng Hospital, Singapore, Singapore. ${ }^{5}$ Department of Cardiology, National University Hospital, Singapore, Singapore. ${ }^{6}$ National Heart Centre Singapore, Singapore, Singapore. ${ }^{7}$ Barts Heart Centre, St Bartholomew's Hospital, London, UK. ${ }^{8}$ The National Institute of Health Research University College London Hospitals Biomedical Research Centre, London, UK. ${ }^{9}$ Cardiovascular and Metabolic Disorders Program, Duke-National University of Singapore, Singapore, Singapore. ${ }^{10}$ Yong Loo Lin School of Medicine, National University Singapore, Singapore, Singapore.
} 
Received: 16 March 2018 Accepted: 9 October 2018

Published online: 08 November 2018

\section{References}

1. O'Gara PT, Kushner FG, Ascheim DD, et al. 2013 ACCF/AHA guideline for the management of ST-elevation myocardial infarction: a report of the American College of Cardiology Foundation/American Heart Association task force on practice guidelines. Circulation. 2013;127(4):e362-425.

2. Ibanez B, James S, Agewall S, et al. 2017 ESC guidelines for the management of acute myocardial infarction in patients presenting with STsegment elevation: the task force for the management of acute myocardial infarction in patients presenting with ST-segment elevation of the European Society of Cardiology (ESC). Eur Heart J. 2018;39(2):119-77.

3. Robinson AA, Jain A, Gentry M, et al. Left ventricular thrombi after STEMI in the primary $\mathrm{PCl}$ era: a systematic review and meta-analysis. Int J Cardiol. 2016;221:554-9.

4. Weinsaft JW, Kim J, Medicherla CB, et al. Echocardiographic algorithm for postmyocardial infarction LV Thrombus: a gatekeeper for Thrombus evaluation by delayed enhancement CMR. J Am Coll Cardiol Img. 2016;9(5):505-15.

5. Moher D, Liberati A, Tetzlaff J, et al. Preferred reporting items for systematic reviews and meta-analyses: the PRISMA statement. Bmj. 2009;339:b2535.

6. Higgins JP, Altman DG, Gotzsche PC, et al. The Cochrane Collaboration's tool for assessing risk of bias in randomised trials. Bmj. 2011;343:d5928.

7. Juni $P$, Witschi $A$, Bloch $R$, et al. The hazards of scoring the quality of clinical trials for meta-analysis. JAMA. 1999;282(11):1054-60.

8. Wallace BC, Dahabreh IJ, Trikalinos TA, et al. Closing the Gap between Methodologists and End-Users: R as a Computational Back-End. J Stat Softw. 2012;49(5):1-15.

9. Gellen B, Biere L, Logeart D, et al. Timing of cardiac magnetic resonance imaging impacts on the detection rate of left ventricular Thrombus after myocardial infarction. J Am Coll Cardiol Img. 2017;11:1404-5.

10. Poss J, Desch S, Eitel C, et al. Left ventricular Thrombus formation after STsegment-elevation myocardial infarction: insights from a cardiac magnetic resonance multicenter study. Circ Cardiovasc Imaging. 2015;8(10):e003417.

11. Lanzillo C, Di Roma M, Sciahbasi A, et al. Cardiac magnetic resonance detection of left ventricular thrombus in acute myocardial infarction. Acute Card Care. 2013;15(1):11-6.

12. Delewi R, Nijveldt R, Hirsch A, et al. Left ventricular thrombus formation after acute myocardial infarction as assessed by cardiovascular magnetic resonance imaging. Eur J Radiol. 2012;81(12):3900-4.

13. Cambronero-Cortinas E, Bonanad C, Monmeneu JV, et al. Incidence, outcomes, and predictors of ventricular Thrombus after Reperfused STsegment-elevation myocardial infarction by using sequential cardiac MR imaging. Radiology. 2017;161898.

14. Biere $L$, Audonnet $M$, Clerfond $G$, et al. First pass perfusion imaging to improve the assessment of left ventricular thrombus following a myocardial infarction. Eur J Radiol. 2016;85(9):1532-7.

15. Weir RA, Martin TN, Petrie CJ, et al. Cardiac and extracardiac abnormalities detected by cardiac magnetic resonance in a post-myocardial infarction cohort. Cardiology. 2009;113(1):1-8.

16. Surder D, Gisler V, Corti R, et al. Thrombus formation in the left ventricle after large myocardial infarction - assessment with cardiac magnetic resonance imaging. Swiss Med Wkly. 2015;145:w14122.

17. Meurin P, Brandao Carreira V, Dumaine R, et al. Incidence, diagnostic methods, and evolution of left ventricular thrombus in patients with anterior myocardial infarction and low left ventricular ejection fraction: a prospective multicenter study. Am Heart J. 2015;170(2):256-62.

18. Kobayashi N, Maehara A, Mintz GS, et al. Usefulness of the left anterior descending artery wrapping around the left ventricular apex to predict adverse clinical outcomes in patients with Anterior Wall ST-segment elevation myocardial infarction (an INFUSE-AMI substudy). Am J Cardiol. 2015;115(10):1389-95.

19. Delewi R, Zijlstra F, Piek JJ. Left ventricular thrombus formation after acute myocardial infarction. Heart. 2012;98(23):1743-9.

20. Mollet NR, Dymarkowski S, Volders W, et al. Visualization of ventricular thrombi with contrast-enhanced magnetic resonance imaging in patients with ischemic heart disease. Circulation. 2002;106(23):2873-6.

21. Srichai $M B$, Junor $C$, Rodriguez $L L$, et al. Clinical, imaging, and pathological characteristics of left ventricular thrombus: a comparison of contrastenhanced magnetic resonance imaging, transthoracic echocardiography, and transesophageal echocardiography with surgical or pathological validation. Am Heart J. 2006;152(1):75-84.

Ready to submit your research? Choose BMC and benefit from:

- fast, convenient online submission

- thorough peer review by experienced researchers in your field

- rapid publication on acceptance

- support for research data, including large and complex data types

- gold Open Access which fosters wider collaboration and increased citations

- maximum visibility for your research: over $100 \mathrm{M}$ website views per year

At BMC, research is always in progress.

Learn more biomedcentral.com/submissions 